\title{
DIFICULTADES GERENCIALES EN FUNCIÓN DEL GÉNERO: CASOS DE EMPRESAS AGROPECUARIAS DE CORRIENTES - ARGENTINA
}

GENDER DIFFICULTIES: CASES OF AGRICULTURAL COMPANIES IN CORRIENTES - ARGENTINA

MARÍA LUDMILA VARGAS VIOLA

FACULTAD DE CIENCIAS EMPRESARIALES UNIVERSIDAD DE LA CUENCA DEL PLATA - UCP

ARGENTINA

PATRICIA LAURA RÁBAGO

FACULTAD DE CIENCIAS ECONÓMICAS UNIVERSIDAD NACIONAL DEL CENTRO - UNICEN

"Si no existe lucha no existe progreso. Aquellos que profesan favorecer la libertad y no obstante desprecian la agitación, son hombres que quieren cosechas sin arar la tierra. Ellos quieren lluvia sin truenos ni relámpagos. Ellos quieren el océano sin el amedrentador rugir de sus aguas". 


\begin{tabular}{rr}
\hline MARÍA LUDMILA VARGAS VIOLA - PATRICIA LAURA RÁBAGO \\
\multicolumn{2}{c}{ DIFICULTADES GERENCIALES EN FUNCIÓN DEL GÉNERO: CASOS DE EMPRESAS AGROPECUARIAS... } \\
Págs. $45-67$ & Recibido: 29/02/2016 \\
\hline & Aceptado: $02 / 06 / 2016$
\end{tabular}

\title{
RESUMEN
}

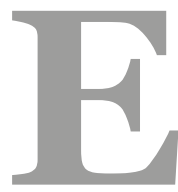

ste trabajo tiene como objetivo analizar la percepción de las dificultades experimentadas por las mujeres propietarias en la gestión de sus empresas rurales en la Provincia de Corrientes, Argentina, y en qué medida han aplicado diferentes estrategias de adaptación o transformación para permanecer en la actividad. Se realizó una investigación cualitativa basada en un estudio de cinco casos, a través de entrevistas personales a mujeres que desempeñan la doble función de propietarias y gerentes de empresas de agropecuarias de mediana y gran escala. Los resultados indican que la principal dificultad es la conciliación entre la vida familiar y profesional, especialmente si tienen niños pequeños. También emergen ciertas limitaciones físicas detectadas en el entorno de trabajo y que en algunas etapas del proceso de producción se pueden percibir como especialmente restrictivas, sobre todo en tareas operativas propias del ejercicio de la actividad de la empresa. Además de los elementos mencionados, las mujeres empresarias no perciben discriminación por su rol asumiendo como naturales las reglas del sector habiendo generado estrategias de adaptación con el fin de superar sus dificultades.

Palabras clave: género; gerentes, dificultades percibidas; empresas rurales.

\begin{abstract}
This paper aims to analyze the perception of difficulties experienced by women owners in the management of their rural enterprises in the Province of Corrientes, Argentina, and if they have applied different adaptive or transformative strategies to remain in activity. It is a qualitative research based on a study of five cases, through personal interviews to women who manage and have companies. The results indicate that the main difficulty is to conciliate family and professional life, especially if they have young-age children. Also, certain physical limitations have been detected in the working environment, which in some stages of the production process may be felt as restrictive, especially in certain operational tasks typical of the exercise of the company's activity. Apart from the mentioned items, women entrepreneurs do not manifest perceiving discrimination on their role, assuming as natural the sector's rules, for which they have generated adaptive strategies in order to overcome their difficulties.
\end{abstract}

Keywords: gender; managers, perceived difficulties; rural enterprises. 


\section{INTRODUCCIÓN}

La presente investigación constituye un estudio de carácter exploratorio buscando determinar las dificultades diferenciales que perciben las mujeres que logran acceder al gerenciamiento de empresas que desarrollan actividades en el sector agropecuario, e indagar acerca de la incidencia tal como sostiene Ferro (2008), que derivarían en la exclusión de facto del patrimonio agrícola a las mujeres. La importancia de identificar estos factores radica en que la igualdad de acceso, incrementaría la productividad de las empresas cuyas propietarias son mujeres (Do, Levchenko \& Raddatz, 2011; Hallward-Driemeier, Hasan, Kamangu, Lobti, \& Blackden, 2013), de forma tal, que el acceso igualitario a recursos productivos podría aumentar el producto (Elborgh-Woytek et al., 2013), asociándose a su vez, la titularidad femenina de explotaciones agropecuarias con una mayor sustentabilidad social y ambiental (Filson, 1993; Traoré, Landry, \& Amara, 1998; Gailhard \& Bojnec, 2015).

La información relevada, da cuenta que en materia de gerenciamiento en el sector agropecuario en la Provincia de Corrientes se encuentra más rezagado que otros sectores económicos en la Argentina, respecto del avance de las mujeres, si bien de acuerdo a los últimos datos disponibles del Censo Nacional de Población y Vivienda (2010) y del Censo Nacional Agropecuario (2008), aunque en este último caso solo fue relevada la mitad de la provincia de Corrientes, sólo un $5 \%$ de los cuentapropistas del sector primario son mujeres. En empresas agropecuarias familiares capitalizadas el gerenciamiento es prácticamente un espacio hegemónico del varón, en tanto, la integración femenina se asocia a roles auxiliares, siendo reconocidas como productoras únicamente, ante la ausencia del esposo o hermano varón, al acceder por viudez, soltería o divorcio (Ferro, 2007). Esta invisibilización y exclusión de la mujer de la producción, limita fuertemente su desarrollo y preparación colocándolas en situación de vulnerabilidad, si deciden o se ven forzadas a asumir la conducción de la empresa agropecuaria familiar.

En general, si bien las empresas familiares latinoamericanas permiten a las mujeres la conciliación del ejercicio profesional auxiliar, con las tareas domésticas, reproductivas y cuidadoras que les son asignadas en forma exclusiva; prevalece en éstas, la cultura patriarcal que cuestiona el ejercicio del liderazgo femenino, fomenta el trabajo invisible y se convierte en un obstáculo que inhibe su desempeño asumiendo únicamente la mujer el rol de líder por defecto (Zabludovsky \& de Avelar, 2001).

Respecto de la actividad agropecuaria específicamente opera un patrón de división del trabajo que desvincula a las mujeres del proceso productivo, asignando al varón aquellos trabajos considerados duros, tales como siembra, cosecha o fumigación, manejo de maquinarias y manejo de ganado; en tanto que, las tareas culturalmente lla-madas "blandas" se asocian a cualidades femeninas (Perona, 2012 a) y hacen que en la práctica, las mujeres sean auxiliares del varón por la compatibilidad de estas tareas con las labores domésticas (Ferber \& Nelson, 2008). Esta desvinculación productiva deriva en un patrón de desvinculación de las mujeres 
de la propiedad rural familiar, que conculca de facto, las normas de herencia igualitaria y opera asignando una jefatura masculina de conducción y titularidad a la explotación agropecuaria familiar, que la mujer solo "posee" a título legal (Ferro, 2008). Adicionalmente las estadísticas públicas, provenientes de los censos nacionales agropecuarios, 2008 y anteriores, refuerzan estereotipos al reconocer únicamente la figura del propietario en lugar de copropietarios, cuando en realidad se trata de explotaciones o empresas familiares.

El objetivo general del presente trabajo es analizar la percepción de eventual existencia de dificultades diferenciales en función del género por las mujeres propietarias-gerentes de empresas agropecuarias familiares radicadas en la Provincia de Corrientes para el ingreso y permanencia en la actividad, y determinar las estrategias adoptadas de superación, clasificándolas en adaptativas o transformadoras.

Constituye un estudio exploratorio descriptivo, llevado a cabo a través de entrevistas semi-estructuradas a cinco casos paradigmáticos de interés intrínseco por el reconocimiento profesional de las entrevistadas, no sólo en Corrientes sino en la región del Nordeste argentino. Los resultados se han validado mediante triangulación metodológica teórica y estudios empíricos realizados para el sector agropecuario en otras regiones del país y América Latina y otros sectores económicos.

El marco conceptual se basa en las dificultades de las mujeres en el ejercicio de su liderazgo y se presenta en el siguiente apartado, finalizado el mismo se explica en detalle la metodología aplicada para obtener los resultados, y la realización de la triangulación a los efectos de validar los mismos, presentándose en la tercera sección los resultados, continuando luego con las conclusiones, donde además se presentan las limitaciones del estudio llevado a cabo.

\section{MARCO CONCEPTUAL}

\section{Dificultades en el ejercicio de la gerencia empresarial}

El liderazgo empresarial desde sus inicios hasta el presente, revela su carácter fuertemente androcéntrico con postulados movidos por los principios básicos de una ética masculina de los negocios, que ha sido dominante en todos los tiempos (García Prince et al., 1993; Ferro et al 2007). En tal sentido, los estereotipos y constructos sociales tienden a asociar ya sea consciente o inconscientemente, a los varones con las características requeridas para el liderazgo y el gerenciamiento (García-Retamero \& López-Zafra, 2006; Schein, 2001). Esta tendencia se refuerza y, en general en diferentes actividades económicas puede observarse bajas tasas de feminización (García-Retamero \& López-Zafra, 2006), lo que podría indicar que las mujeres parten en desventaja para el ejercicio del liderazgo y de puestos de decisión.

Estas dificultades diferenciales de las mujeres en los puestos gerenciales se han simboli- 
zado mediante representaciones simbólicas o metafóricas, tales como "El Muro", "El Techo de Cristal”, "El Laberinto" y "El precipicio de Cristal. Así, "El Muro" se utilizó a mediados del siglo pasado para indicar la existencia de dos áreas diferenciadas; la esfera pública y productiva, considerada como reservada a los varones; y la esfera reproductiva, privada y doméstica de dominio de las mujeres, observándose que cada una de estas áreas están basadas en supuestos conductuales determinados por la biología y las tradiciones que se asumieron inherentes a cada sexo. De modo que la capacidad de procreación de las mujeres y las ventajas comparativas en cuanto a capital invertido y transmitido por generaciones en destrezas en el ámbito doméstico y en entornos semipúblicos, las convertiría en más aptas para la realización éstas tareas, lo que las sitúa en desventaja para la realización de otras tareas, (Becker \& de Grado, 1987). El limitado número de mujeres que alcanzó a detentar posiciones de liderazgo y poder en espacios masculinos no fue cuestionado por este ejercicio ya que lo había logrado por mérito propio ejerciendo liderazgos tradicionales y adaptativos (Eagly \& Carli, 2003a). La segunda y quizá la más famosa es la metáfora de "El Techo de Cristal" (Bryant, 1984;Hymowitz \& Schellhardt, 1986) utilizada para describir la existencia de un límite invisible que impide el ascenso de la mujer a cargos gerenciales y puestos de mando aun cuando las calificaciones académicas y laborales fueran equivalentes a las de los varones. A partir de una relativa inserción de las mujeres en posiciones de poder surge una nueva metáfora, "El Laberinto o El Laberinto de Cristal" (Barberá, Ramos \& Candela, 2011), en alusión a la existencia de las mayores dificultades que deben enfrentar las mujeres para detentar posiciones de poder, pero que resultan franqueables a aquéllas que encuentran las salidas con talento, perseverancia, suerte o a quiénes sean conducidas por mentores o padrinos por los caminos del laberinto (Eagly \& Carli, 2003b). Éste es un punto de inflexión que resulta relevante ya que las investigaciones no solo se basan en dificultades, sino que además comienzan a explorarse "atributos, factores o características" facilitadoras para el liderazgo.

Finalmente, uno de los últimos términos acuñados es "El precipicio de Cristal" y evidencia la asignación a las mujeres de puestos nóveles, de bajo presupuesto, que pueden ser considerados prescindibles en un inicio, pues el objeto de los mismos es cumplir con "los cupos femeninos" establecidos institucionalmente, en los cuales, las posibilidades de fracaso son superiores a la media o son aquellos que poseen mayores exigencias de resultados (Ryan \& Haslam, 2005, 2008), tanto en el ámbito gerencial empresario como en el ámbito político.

En estas dificultades diferenciales respecto de los varones que han sido denominadas "barreras" existen una serie de dimensiones enunciadas empíricamente, las cuales pueden agruparse en barreras psicológicas o personales, sociológicas, organizacionales y normativas.

Entre las barreras psicológicas o personales se destacan la ausencia de motivación en las mujeres, influyendo en la personalidad o la existencia de conflictos personales debido a la doble responsabilidad por las actividades domésticas y laborales (Ramos et al, 2002), argumentándose también que este conflicto personal es subyacente por un lado, a la socialización primaria recibida y por otro, al aislamiento social y afectivo de aquellas mujeres exitosas, re- 
sultado en una amenaza potencial para sus parejas (Guirado et al, 2006), incluso pudiéndose dar situaciones de odio o emulación de otras mujeres no exitosas (Echaniz, 2001), o en otros casos presentar falta de apoyo familiar (Lupano, 2009), ocasionando conflictos personales por la responsabilidad doméstica (Ferro, 2007).

Las barreras sociológicas se presentan a menudo por el conflicto socialmente naturalizado entre los roles doméstico de cuidadora dentro del hogar asignado exclusivamente a las mujeres (Provoste Fernández, P. 2012) y el vínculo existente entre la función reproductiva con el rol de profesional (Ramos et al, 2003), aunque también se describen casos de discriminación desde un fenómeno histórico y cultural (Guirado et al, 2006); a lo que debe adicionarse prejuicios basados en estereotipos y preconceptos (Lupano,2009), dando lugar a situaciones de elegir la promoción de los varones ante la posibilidad de maternidad de las mujeres (Gatica \& Rojas, 2004; Pérez et al, 2004; Heller, 2004). En el caso específico de estudios rurales se argumenta que suele transmitirse en forma consuetudinaria la administración del patrimonio femenino por el cónyuge varón (Ferro, 2007) efectuándose incluso el alquiler de la propiedad rural familiar femenina a familiares varones a valores que resultan inferiores a los del mercado (Paulilo, 2006). También existe una identificación de brechas entre la legislación que garantiza herencia igualitaria y los bienes que se otorgan de hecho (en la práctica); indicando diferencia en el reparto de los bienes que opera en detrimento de las hermanas mujeres (Archetti et al, 1977; Bourdieu, 2000; Preda, 2010).

Dentro de las barreras organizacionales no solo pueden encontrarse estudios que enfatizan sobre cuestiones actitudinales y demográficas en las organizaciones, sino a ciertos ambientes que se presentan hostiles para las mujeres (Ramos et al, 2002); señalando a su vez cierta incongruencia de los roles de género con la gerencia (Schein, 2001; García \& López, 2006; Haslam \& Ryan, 2008; Godoy \& Mladinic, 2009; Ramos, et al 2002). Desde este punto de vista se analiza y observa el contexto organizacional en el que se desempeñan los individuos (Guirado et al, 2006), en el cual suele presentarse como conclusión que dentro de las organizaciones, los otros gerentes no están acostumbrados a mujeres en puestos gerenciales (Fernández et al, 2012), encontrándose a su vez excluidas de los canales informales del mundo empresario (Lupano, 2009), existiendo en algunos casos ciertas estrategias indirectas de comunicación en el caso de los pedidos femeninos (Babcock \& Laschever, 2009), presentándose una invisibilización de las mujeres productoras (Gasson \& Winter, 1992; Navarro, 1999; Perona, 2012b).

En las barreras normativas, se encuentran agrupadas aquellas prácticas normativas del Estado que reproducen desigualdad, como en el caso de las estadísticas oficiales que no identifican a las copropietarias mujeres en establecimientos rurales (Guirado, Alonso \& Luque, 2006), pudiéndose observar también en la actualidad, las prácticas en las que se privan de los medios de producción a mujeres (Do et al, 2011), bajo diferentes modalidades. 


\section{METODOLOGÍA}

El presente trabajo se emplea una investigación de tipo cualitativa, exploratoria descriptiva no experimental, sobre una población de mujeres que desempeñan el rol de gerentes en empresas agropecuarias en Corrientes (Argentina). Las unidades de análisis se determinaron mediante un muestreo intencional de casos, dado que fueron seleccionados por tratarse de mujeres, propietarias o copropietarias en ejercicio de la gerencia en empresas agropecuarias, localizadas en Corrientes (Argentina), reconocidas socialmente por la sostenibilidad económica y financiera de sus empresas. El tamaño de explotación elegido, superior a 1500 hectáreas se debe a que el mismo constituye la unidad de explotación económica viable en la provincia donde se realiza el estudio para la actividad de cría, considerándose a precios corrientes y calculando un nivel destete promedio del 60\% (Altamirano et al 2009).

La recolección de datos fue realizada mediante entrevistas personales, formales autobiográficas, individuales, grabadas a cinco mujeres, durante el año 2013. Utilizándose guías de entrevistas con preguntas abiertas y preguntas cerradas. Esta metodología, se considera pertinente debido a que el problema a indagar es un fenómeno multidimensional que requiere recabar la vida, experiencias, ideas, valores, dificultades y representaciones de la estructura simbólica de las entrevistadas desde su propia perspectiva contextualizada, de modo de permitir a la vez la comunicación y la construcción conjunta de significados. Durante las entrevistas, se ficharon comentarios, aspectos ambientales, actitudinales y lenguaje gestual, matices diálogo, silencios, énfasis y entonaciones.

El análisis de datos se efectuó a partir de la desgrabación de entrevistas y organización de áreas emergentes de los ejes de entrevistas, tales como 1) Datos personales, caracterización de la entrevistada y su núcleo familiar; 2) Caracterización de la empresa, explotaciones, procesos productivos actuales y pasados desarrollados; 3) Dificultades gerenciales y personales percibidas en función del género en distintas fechas de su ejercicio profesional y 4) Estrategias aplicadas para la superación de eventuales dificultades y rutinas de trabajo.

Las transcripciones se ordenaron a tres columnas y se codificaron estableciendo subcategorías, categorías y meta-categorías a partir del marco referencial descripto. El análisis e interpretación del discurso se realizó a la luz de categorías y dimensiones de partida y de las emergentes del trabajo de campo, siguiendo el método de comparación constante, combinando la generación inductiva de categoría con una comparación simultánea de todos los incidentes observados, los cuales fueron comparados además con los resultados de otras investigaciones empíricas para ir refinando los procesos de categorización (Creswell, 2003). En tal sentido, se seleccionaron citas o incidentes para asignarles un código que permitiera además de conformar la categoría; utilizarla para efectuar consistencia. Finalmente, se regresó sobre las entrevistadas para que manifestaran su opinión sobre los resultados y ofrecieran alguna información adicional que consideraran oportuna. 


\section{RESULTADOS}

A partir del análisis de los datos obtenidos en las entrevistas efectuadas se presentan las características generales de las empresas, el perfil de las entrevistadas y la percepción que tienen las entrevistadas respecto de la posible existencia de barreras identificadas en la literatura especializada.

\subsection{Características generales productivas de las empresas relevadas}

Las cinco empresas gerenciadas por mujeres pueden caracterizarse como empresas familiares, de más de dos generaciones en la actividad pecuaria y guardan similitudes en cuanto a estructura, características, relaciones con el contexto socioeconómico del medio rural y tecnología utilizada, que se sintetizan en la tabla 1. El tamaño medio es de aproximadamente 9.00o hectáreas distribuidas entre una y cinco explotaciones (propias o afectadas bajo contrato de arrendamiento). Las superficies resultan superiores a las de los modelos "farmers" de la región centro y pampeana del país, por tratarse de campos pecuarios de dos ciclos o de ciclo completo.

Tabla 1: Modelos productivos gerenciados por mujeres en la Provincia de Corrientes.

\begin{tabular}{|c|c|c|c|c|c|}
\hline $\begin{array}{l}\text { Categoría } \\
\text { relevada }\end{array}$ & Empresa 1 & Empresa 2 & Empresa 3 & Empresa 4 & Empresa 5 \\
\hline $\begin{array}{c}\text { Antigüedad } \\
\text { empresa } \\
\text { (generaciones) }\end{array}$ & cinco en la familia & tres en la familia & dos en la familia & cuatro en la familia & dos en la familia \\
\hline $\begin{array}{c}\text { Superficie } \\
\text { explotada (Has) }\end{array}$ & 8.270 & 9.000 & 5.200 & 3.700 & 17.400 \\
\hline $\begin{array}{l}\text { Cantidad de } \\
\text { explotaciones } \\
\text { que integran } \\
\text { la empresa }\end{array}$ & 5 & 5 & 2 & 1 & 2 \\
\hline $\begin{array}{l}\text { Actividades } \\
\text { productivas }\end{array}$ & $\begin{array}{l}\text { Ganadería ciclo } \\
\text { completo y agricultura } \\
\text { para engorde ganado }\end{array}$ & $\begin{array}{l}\text { Ganadería ciclo } \\
\text { completo y agricultura } \\
\text { para engor de ganado }\end{array}$ & $\begin{array}{c}\text { cabaña, cría } \\
\text { y recría }\end{array}$ & $\begin{array}{c}\text { cabaña, cría, recría, } \\
\text { producción arrocera } \\
\text { y producción de } \\
\text { árboles frutales }\end{array}$ & $\begin{array}{c}\text { cría } \\
\text { y recría }\end{array}$ \\
\hline $\begin{array}{l}\text { Porcentaje de la } \\
\text { facturación } \\
\text { proveniente } \\
\text { de la ganadería }\end{array}$ & $100 \%$ & $100 \%$ & $100 \%$ & $80 \%$ & $100 \%$ \\
\hline $\begin{array}{c}\text { Cantidad de } \\
\text { personal }\end{array}$ & 17 & 10 & 10 & 5 & 22 \\
\hline
\end{tabular}

Fuente: elaboración propia con base a datos de entrevistas 
De los testimonios surge además que los objetivos empresarios en cuanto a rentabilidad, conservación, en diferentes contextos macro y microeconómicos y expansión en el mediano y largo plazo, que coinciden en las cinco explotaciones. En las actividades de cría y recría, manifiestan la tendencia a la incorporación de capital, tecnología y conocimientos, a través de pasturas y verdeos de invierno, riego y aguadas y la realización de potreros con el manejo de técnicas tales como el equilibrio de rodeos, inseminación a tiempo fijo, suplementación animal y genética, utilizando razas británicas (Hereford y Aberdeen Angus) en cruzamiento con índicas (Brahaman) pudiéndose apreciar una tendencia a la especialización de la cadena de producción en cada una de las explotaciones o campos que conforman la unidad de negocio. La estrategia de producción se orienta al aumento de la carga promedio por hectárea, y en dos casos se han relevado actividades de cabaña, en tanto que en la explotación de menor tamaño se realiza un manejo diversificado intensivo que incluye producción arrocera, frutihortícola y eslabonamiento de la cadena productiva de carne hacia campos especializados en la pampa húmeda. En cuatro de los cinco casos, la ganadería aporta el cien por ciento de la facturación, en la estructura diversificada se reduce al $80 \%$. Todas las empresas han sido transferidas de generación en generación, no obstante como consecuencia de la baja tasa de rentabilidad de la actividad respecto del costo de la tierra del quinquenio precedente, la expansión de las empresas se ha dado mediante un cambio en el modelo de negocios que opera mediante incorporación de explotaciones por vía de arrendamiento que se incorporan marginal y/o estacionalmente.

Únicamente la empresa representada en el caso 4, legalmente es considerada como una empresa unipersonal, en las restantes, el capital se organiza en acciones; señalando las entrevistadas que esta estructuración resulta ventajosa para organizar la sucesión.

\subsection{Perfil de las entrevistadas}

Como puede observarse en la Tabla 2, la edad promedio es de 58 años, con doce años promedio al frente de la gerencia, contando con experiencia y capacitación específica en el área, en algunos casos mediante estudios formales y en otros con distintos tipos de cursos realizados al efecto. Las cinco entrevistadas son madres y tres de las cinco están actualmente casadas. Cuatro de las cinco entrevistadas ingresaron a la actividad por herencia de tierras y/o estructuras productivas pecuarias ante el fallecimiento del padre y sólo una de ellas accede a partir del fallecimiento del esposo, contando en ese momento con hijos menores. 
Tabla 2: Caracterización de las entrevistadas

\begin{tabular}{|c|c|c|c|c|c|}
\hline $\begin{array}{c}\text { Categoría } \\
\text { relevada }\end{array}$ & Entrevistada 1 & Entrevistada 2 & Entrevistada 3 & Entrevistada 4 & Entrevistada 5 \\
\hline Edad & 62 & 62 & 66 & 40 & 57 \\
\hline $\begin{array}{c}\text { Antigüedad en el } \\
\text { gerenciamiento } \\
\text { en años }\end{array}$ & 17 & 14 & 14 & 8 & 8 \\
\hline Acceso & $\begin{array}{c}\text { Herencia del } \\
\text { padre }\end{array}$ & $\begin{array}{c}\text { Herencia del } \\
\text { padre }\end{array}$ & $\begin{array}{c}\text { A partir del } \\
\text { cónyuge }\end{array}$ & $\begin{array}{c}\text { Herencia del } \\
\text { padre }\end{array}$ & $\begin{array}{c}\text { Herencia del } \\
\text { padre }\end{array}$ \\
\hline $\begin{array}{c}\text { Máximo nivel } \\
\text { académico } \\
\text { alcanzado }\end{array}$ & $\begin{array}{c}\text { Licenciada en } \\
\text { Administración }\end{array}$ & $\begin{array}{c}\text { Secundario } \\
\text { completo }\end{array}$ & Perito & $\begin{array}{c}\text { Licenciada en } \\
\text { economía } \\
\text { agropecuaria }\end{array}$ & $\begin{array}{c}\text { Profesora y técni- } \\
\text { ca en empresa } \\
\text { agropecuaria }\end{array}$ \\
\hline Cantidad de Hijos & 4 & 3 & 2 & 2 & 3 \\
\hline Estado civil & Divorciada & Casada & Viuda & Casada & Casada \\
\hline
\end{tabular}

Fuente: elaboración propia con base a datos de entrevistas

Todas las entrevistadas manifiestan haber permanecido en la actividad, sin planear esta carrera, y que ejercían con anterioridad otras profesiones compatibles con el rol doméstico, no obstante, han elegido permanecer a partir de la percepción de la tierra y de las explotaciones (generalmente con nombre femenino) como una parte integrante e indivisible de la familia y el aliento de padres y/o esposos para continuar en la actividad familiar.

En el momento de hacerse cargo de las empresas manifiestan haberse planteado como único objetivo mantener económicamente a la familia y poder conservar al menos la tierra, no obstante, las empresas han crecido respecto a las que recibieron en términos de facturación, infraestructura, instalaciones y genética, habiendo en algunos casos podido anexar tierras, por lo que en la autoevaluación de su gestión se consideran exitosas, aunque respecto de la asunción de riesgo en los negocios consideran que tienen un perfil mas bien conservador quizá dado por un mandato familiar tácito dado que provienen de familias tradicionalmente productoras pecuarias y eventualmente agrícolas.

Además durante las entrevistas, se refieren a la explotación original por el nombre femenino del establecimiento como si se estuvieran describiendo a una integrante más de la familia.

Entrevistada 1:... "La (nombre de la explotación) ha pertenecido a mi familia por cinco generaciones, cuando me hice cargo [hace una pausa; rememora]... mi objetivo fue por lo menos poder mantener la tierra".

Entrevistada 2:... "No he salido de la actividad por tradición y aunque sea mujer; he querido conservar por una cuestión de apego y tradición. Mis hijas 
no están en esto ...Pero ellas aman "La (nombre de la explotación)" [énfasis de la entrevistada].

Entrevistada 5: .. “.. Y mirá;.. Estoy en conexión con esto desde que nací, aunque antes no me dedicara, como ahora, pero está en mi sangre desde siempre”.

Como puede apreciarse en los dichos de las entrevistadas, estas explotaciones agropecuarias no son sólo fuente de sustento familiar o un factor de la producción, sino que constituyen un modo de vida que determina una pertenencia social a un grupo con características diferenciadas, con fuerte raigambre en las tradiciones y el mandato de conservación de la tierra.

\subsection{Dificultades o Barreras}

Las dificultades a las que se enfrentan las entrevistadas pueden observarse tanto en el plano familiar como en el rol gerencial en una actividad en la que tradicionalmente se inclina por el desempeño del varón, en tal sentido se exponen a continuación las barreras percibidas por las entrevistadas.

\subsubsection{Barreras Personales o Psicológicas}

Estas barreras son percibidas a partir del conflicto entre la relación en la vida familiar y su dedicación a la empresa familiar como así también en cuanto a los conocimientos necesarios en el tipo de actividad específica de cada establecimiento.

\subsubsection{Conflicto Familia - Trabajo}

En la mayoría de este tipo de explotación, es habitual que la actividad se desarrolle con un sesgo orientado en mayor medida hacia la producción que la gestión, por tal motivo existen ciertas costumbres como el asentamiento de la familia en el lugar en que se lleva a cabo la explotación, pudiendo originarse la existencia de conflictos de acuerdo a la etapa familiar por la que van transitando las entrevistadas. En tal sentido, de las cinco entrevistadas, tres de ellas no residen en la explotación y lo hacen en centros urbanos cercanos debido a la falta de acceso a servicios de salud y educación en áreas rurales de Corrientes y su relativa proximidad con las explotaciones (menos de 100 kilómetros en promedio), no obstante puede observarse que no constituye un conflicto para las mismas, dado que mas bien se presenta como un cambio en las tradiciones.

Entrevistada 1: "la verdad nunca me planteé vivir en el campo, es cerca, ... y la verdad: no vivo en el campo porque no me gusta esa vida de campo, faltan servicios de comunicación y existen dificultades para la crianza de los hijos y su escolarización. Considero que radicarse en el campo resulta imposible si se pretende que los hijos accedan a servicios de salud y educación de calidad.... Aún 
hoy (énfasis de la entrevistada) para poder hablar por teléfono tengo que subir al techo con el celular y sujetarme de una rama, imagínate diez años atrás”. Entrevistada 2.... "la maternidad dificulta el ejercicio de la gerencia por una cuestión de tiempo y que en el campo no se cuenta con servicios de salud y educación de calidad, hoy no tengo esas dificultades porque ya mis hijas son grandes".

Respecto de las dos entrevistadas que residen en la misma explotación que gestionan, una de ellas tiene hijos adultos y reconoce que por su edad y la de sus hijos está en una situación privilegiada para asignarle tiempo al ejercicio de la actividad; que antes hubieran significado sacrificios en términos de la vida familiar.

Entrevistada 3: "La edad en la que me hice cargo fue una fortaleza porque mis hijos ya estaban casados, tengo independencia total de horarios [énfasis de la entrevistada], no valoro mi tiempo libre, es decir, me quedo leyendo a cualquier hora, siempre hago esto".

Finalmente, la entrevistada con hijos en edad escolar, manifiesta haber adoptado un conjunto de estrategias consistentes en el agrupamiento de varias actividades extracurriculares a sólo dos jornadas semanales en las que reduce la jornada laboral en el campo y la instrucción personal de sus hijos en el hogar a través de ejercicios adicionales que ella misma imparte y supervisa en defensa de su actividad laboral.

Entrevistada 4: Normalmente ser mamá entra en conflicto con el ejercicio de la gerencia agropecuaria, no estoy de acuerdo que radicarse en el campo obstaculice la educación sino por el contrario te posibilita concentrarse al interior de la familia en la escolarización primaria, que seguro es de más calidad que la del pueblo, obvio (énfasis de la entrevistada).

... Es un poco dificil, porque se cumplen roles, de funcionamiento de una casa, de madre, de empresaria y dentro de empresaria, manejo diario de la administración, comercialización, todo, ...aunque...[pausa] siempre tuve en claro la parte de los hijos (poniendo énfasis la entrevistada respecto de la crianza de los mismos) (Énfasis de la entrevistada) La mujer tiene limitaciones en el estar por la maternidad y otras responsabilidades que tiene la mujer que no las tiene el hombre.

\subsubsection{Conflictos en la actividad productiva}

Tradicionalmente, en los puestos directivos de la actividad agropecuaria el lugar ocupado por mujeres ha sido escaso, dando lugar a que surja en el imaginario colectivo una creencia respecto de que los varones están mejor preparados que ellas, surgiendo así cierto compromiso adi- 
cional en capacitación específica respecto de la actividad, aunque no necesariamente en capacitación gerencial. En tal sentido, tres de las cinco entrevistadas, consideran que poseen un menor conocimiento de los aspectos productivos de la actividad como debilidad a sortear respecto de pares varones, manifestando cierto conflicto personal al percibir un menor conocimiento al respecto, coincidiendo en aquellas entrevistadas que tienen una mayor desvinculación productiva las actividades rurales cotidianas, lo cual puede interpretarse como causa y consecuencia en un círculo que se reproduce. Las entrevistadas que buscan superar esta falencia con capacitación, lectura y asesoramiento externo, lo indican como se expone a continuación.

Entrevistada 1: ..."Conozco el negocio lo suficiente como para manejar la empresa, pero creo que se menos que un hombre...”.

Entrevistada 2: ..."Me faltaría conocer más de la parte productiva, traslados, ventas, etc.". ... "No me gusta tomar decisiones en forma unipersonal de la parte productiva".

Entrevistada 5: "Después de mi carrera; cuando tuve que hacerme cargo de esto estudié un terciario de gestión de empresas agropecuarias, para aprender más; me sirvió.

Por otro lado, las mujeres que participan activamente de las tareas productivas, no perciben escaso conocimiento en el tema, declarándose a la vanguardia en la actividad, a la vez que dan cuenta de un conocimiento superior, respecto de las que no están involucradas directamente e incluso al de sus pares varones, considerándose respaldadas por un proceso de formación continua y asesoramiento externo.

Entrevista 3: "Leo bastante....Tomo decisiones en forma personal, en la parte productiva. Antes, decidía con mi asesor que ha fallecido y a quién no le encuentro aún sustituto, como lo extraño al Doctor!. Siempre he tenido halagos de mis pares, fui la primera en hacer destete, me invitaron a dar charlas, te tiene que gustar; uno pone empeño y dedicación”.

Entrevista 4: "Conozco el negocio más que cualquiera, veo y siento que todo funciona de primera cuando yo estoy, ........hay cosas y actividades que me arriesgo a hacer porque estoy acá para controlar".

\subsubsection{Barreras Sociológicas}

Estas mujeres se enfrentan en determinado momento de su vida al hacerse cargo de los negocios familiares en una situación de conflictividad con la sociedad ante ese rol que le adjudican las costumbres de la sociedad en la que se criaron y el mandato recibido y las nuevas funciones desarrolladas. 
Las cinco entrevistadas no hablan en reuniones sociales con pares mujeres o varones respecto de la actividad y no consideran a este tipo de conversaciones pertinentes; exteriorizan que los hombres son más propensos a intercambiar este tipo de información en reuniones sociales.

Entrevista 3... "De lo que hago en el campo solo lo hablo con mis hijos y en reuniones sociales me adapto a temas de mujeres...”.

No obstante, consideran que la discriminación a la que están expuestas es menor que en otras actividades por la existencia de círculos cerrados, existe pleno conocimiento de los vecinos, proveedores, comisionistas y compradores, asesores, otros. Las relaciones son sostenidas en el tiempo y el personal de campo, regularmente es continuador de sus antecesores. Las mujeres señalan que no obstante, pueden elegir con quién vincularse en función del trato que reciban.

Entrevistada 4: "si, hasta que te conocen; al principio todos creen que no conoces del negocio y obvio, [pausa y énfasis].

Entrevistada 5: "La persona a cargo de la empresa agropecuaria se mueve en un círculo no tan grande, es más fácil que una gerente de una empresa que no sea del rubro agropecuario, a mi amiga que es súper capaz, no le dan la gerencia por ser mujer, acá esto no pasa, además claro, entrás como la dueña, no te van a cuestionar o al menos no en la cara. La gente es respetuosa, después no sé qué dirán.

\subsubsection{Barreras organizacionales}

Otro tipo de barreras que aparecen en el ejercicio de la actividad están relacionadas específicamente con la conflictividad en el ejercicio de la gestión y pueden estar relacionadas a limitaciones físicas, en cuanto al tipo de actividad, o por el lenguaje utilizado, dado que es considerada una actividad fundamentalmente masculina, o bien por los canales de comunicación con el entorno o con sus subordinados, tal como se presentan en los apartados siguientes.

\subsubsection{Conflictos por ambientes hostiles de trabajo}

Como puede observarse en los dichos presentados a continuación, esas limitaciones físicas propias de la actividad productiva, también afectan a las entrevistadas.

Entrevistada 1: "Surgen también las limitaciones fisicas para hacer frente a los extremos climáticos y las largas jornadas".

Entrevistada 3: "La verdad, existe, una limitante física en el trabajo, principalmente en lo que son las tareas operativas, pero yo me ocupo personalmente, aunque las tareas están divididas y asignadas las responsabilidades”. 
Entrevistada 4: Mirá...yo cuando me hice cargo hombreaba bolsas [énfasis de la entrevistada], todo es maña no fuerza...y la gente habrá pensado, mira, descargando el camión, con qué necesidad...; hoy pienso, con qué necesidad, pero no me arrepiento, la verdad tenía que armar la empresa porque sólo heredé la tierra y alguna hacienda.

3.3.3.2. El sexismo en el lenguaje y la identificación masculina del espacio

Tres entrevistadas pusieron de manifiesto la incomodidad que generan las costumbres de un uso de lenguaje propio del ethos masculino, en particular en las tareas específicas de la actividad pecuaria en la realización de tacto y castración a los animales. En este punto, la mayoría de las entrevistadas acepta esta situación y opta por estrategias adaptativas como la delegación en terceros de estas actividades productivas:

Entrevistada 1: "estar en el campo requiere un sexo masculino, todos son hombres, la comunicación rural de los corrales es para hombres".

No obstante, una de las entrevistadas desnaturaliza esta construcción cultural y expresa haber indicado a sus subordinados pautas de lenguaje en corrales, lo que constituye una estrategia transformadora deliberada. Las normas conductuales, les son informadas personalmente a sus dependientes al inicio de la relación laboral, habiendo construido para sí misma su propio espacio de trabajo.

Entrevistada 3: "Al personal, siempre le explico que acá las cosas son diferentes, la cabeza es una señora y así es la cosa, la señora [habla de sí misma en tercera persona] puso ciertos códigos en la comunicación, en el trabajo en el corral, acá es distinto el lenguaje porque está la señora [énfasis de la entrevistada] a los animales les decimos,[gesticulando con sus manos como si tuviera una vara] "vamos niñas y vamos niños".

\section{3·3.3.3. Dificultades en la comunicación con subordinados}

Si bien la medida del éxito de una gestión puede ser de tipo subjetivo, la comunicación en una organización es uno de los elementos fundamentales para la transmisión de los objetivos, la explicitación de las actividades y la forma en que se pueden obtener los resultados, por tal motivo es deseable que una comunicación sencilla y natural. En tal sentido, sólo una de las entrevistadas manifiesta dificultades en la comunicación con los subordinados:

Entrevistada 4: El manejo diario es difícil y cada vez se pone más difícil con el personal, por la falta de responsabilidad, se perdió la cultura de trabajar, es 
necesario conocer muy bien los trabajos diarios para poder mandar, llevarlos adelante, como y el tema de manejar el personal es complicado, hay que estar a diario, y marcar el paso, ahí, todos los días, sin dudar...”.

Las restantes entrevistadas, si bien señalan no tener dificultades, tienden a disimular, disfrazar o mostrar una cara distinta de la autoridad y establecer nexos en la comunicación:

Entrevistada 3: apenas entra alguien al campo, me siento con la persona mano a mano y le explico las cosas como son...".

\section{3·3.3.4. Exclusión de los canales informales del mundo empresario}

Los roles de género condicionan también los intercambios informales de información con pares, para subsanar esta situación dos de ellas participan de un grupo denominado Consorcio Regional de Experimentación Agrícola루, -CREA-; (donde los intercambios de información están pautados y organizados) y una tercera entrevistada forma parte de la conducción gremial de la Sociedad Rural local ${ }^{2}$.

Entrevistada 1: ..."los hombres tienen mayores conocimientos de que hacen los otros y cómo les va, qué tiene cada uno y qué tenés vos, yo no pregunto y no hablo frecuentemente de la actividad".

Entrevistada 2: "Ellos, están al tanto de que hace cada uno en el sector, se cuentan, comentan, se miden y te miden".

Entrevistada 4: ..."Donde más me entero que hace el resto, (en referencia a otros productores), es en las reuniones de CREA, ahí la información fluye, además te cuentan que hace el resto, no tenés que preguntar, basta sólo escuchar”.

\subsubsection{El entorno y la inseguridad}

Los delitos con móviles económicos contra propiedades y personas, constituyen una dificultad extra, y que resulta frecuente para estas mujeres, cuyo riesgo aumenta cuando deben desplazarse en soledad con niños y en caminos rurales, dado que los mismos suelen ser oscuros e intransitables en caso de lluvias o sequías extremas. Las entrevistadas no son ajenas a las dificultades generales por las que atraviesa la región, y si bien las del entorno pueden sobrellevarlas en forma más tranquilas, tales como la falta de infraestructura de rutas y caminos, también han experimentado los problemas derivados de la falta de seguridad, tal como se ejemplifica a continuación.

\footnotetext{
${ }^{1}$ También conocido como Movimiento CREA que reúne a 2032 empresas agropecuarias que a través del intercambio de ideas y experiencias consideran que pueden mejorar los resultados de sus organizaciones, los cuales están nucleados en la Asociación Argentina de Consorcios Regionales de Experimentación Agrícola (AACREA), una organización civil sin fines de lucro.

${ }^{2}$ La Sociedad Rural Argentina es una entidad gremial patronal del sector agropecuario.
} 
Entrevistada 4: "Una noche cuando venía con los chicos me asaltaron en la portada, me estaban esperando (...).

No obstante no deja de ser tomado en forma anecdótica y seguir con sus actividades habituales para conseguir sus objetivos planteados en la gestión de sus empresas.

\section{CONCLUSIONES}

Si bien en los últimos años existen mayores experiencias de mujeres en el ejercicio de la gerencia, la literatura especializada enumera una serie de dificultades a las que se enfrentan en su carrera profesional. Teniendo en cuenta esas barreras, el presente trabajo se planteó como objetivo analizar la percepción de la existencia de dificultades diferenciales en función de género por aquellas propietarias que cumpliendo su rol de gerentes en empresas agropecuarias familiares las han detectado en el ingreso o permanencia en la actividad y en ese caso cuáles fueron las estrategias adoptadas.

En las entrevistas realizadas a las cinco propietarias - gerentes, se puede apreciar que las explotaciones guardan similitudes entre sí en cuanto a estructura, características, relaciones con el contexto socioeconómico del medio rural y tecnología utilizada, conformando empresas caracterizadas como familiares, cuya actividad principal es de tipo agropecuaria. Las mujeres ingresaron a la actividad luego del fallecimiento del padre o esposo, permaneciendo en la misma por la existencia de un "mandato familiar" de conservar la tierra para las generaciones futuras. En tal sentido, la "tierra o explotación agropecuaria originaria" representa algo más que un factor de la producción, pues tiene un alto valor afectivo, pudiéndose observar que las entrevistadas utilizan el nombre propio de las estancias, las cuales tienen nombre de mujer.

Las cinco empresarias coinciden en señalar que sus gerencias han sido exitosas ya que superaron ampliamente sus propias expectativas, habiendo podido vivir de la rentabilidad empresarial, conservar la tierra y crecer en producción, atravesando tanto ciclos de conservación, como de expansión conforme al contexto macroeconómico y las políticas públicas destinadas al sector.

Las empresarias entrevistadas no manifiestan una clara alineación con la evolución de las diferentes teorías de género, aunque a partir de la cantidad de establecimientos gestionados por estas mujeres y la forma de acceso a la gestión de los mismos, la feminización en relación con otros sectores productivos está rezagada pudiendo asimilarse la inserción de la mujer a la metáfora "El Muro", lo que explicaría la falta de discriminación percibida hacia el grupo entrevistado que ha logrado atravesarlo, situación que podría verse reforzada a partir de haber llegado no por haber buscado ex profeso ese rol, sino por haber ocupado sus lugares a partir de una continuidad en la actividad familiar. Si bien todas han exteriorizado que el ser mujer no 
es una dificultad adicional, acuerdan al señalar que les hubiera sido más fácil llevar adelante sus funciones de haber sido varones. Esta percepción de mayores esfuerzos en relación a los pares varones es compartida por las gerentes de la mayor parte de las actividades económicas y líderes de todos los sectores. La aparente contradicción que se desprende de lo anterior, debe entenderse aquí como la negación para sí mismas de dificultades que claramente resultarán existentes para otras. Este grupo de mujeres, en general, asume como natural las reglas del sector, generando estrategias adaptativas en pos de la superación de las dificultades diferenciales percibidas y a su vez, habiendo recibido la formación de sus padres, mantienen vigentes las tradiciones propias del sector. Sólo una de las entrevistadas (que no fue formada por el padre) manifiesta la adopción de estrategias deliberadas transformadoras del ambiente.

Las dificultades en torno a la conciliación de las actividades familiares, maternidad y organización familiar, (actividades que son consideradas tradicionalmente exclusivas y excluyentes de la mujer) con las profesionales se intensifican en los entornos rurales de la Provincia de Corrientes, debido a la falta de acceso a bienes sociales y a las nuevas expectativas de consumo y educación cuyos proveedores se concentran en los centros urbanos. Las entrevistadas perciben barreras personales tales como las descriptas en otros estudios en cuanto a la existencia de doble responsabilidad entre su actividad laboral y el rol doméstico, pues en general la primera ha sido organizada en función de las etapas por las que atraviesan sus hijos, generando tensiones para la conciliación de la actividad influenciada por la falta de servicios diferenciados que pretenden para su grupo familiar, tales como educación y salud, en conjunción con la ubicación del establecimiento al estar alejado de centros urbanos. La unidad de producción agrícola queda en el campo y existe un desplazamiento dificultado para mujeres.

Las dificultades organizacionales no difieren de otros espacios marcadamente masculinos, destacándose los extremos climáticos, la inseguridad y el lenguaje como dificultades no categorizadas en otros sectores. Sin embargo, el conjunto de dificultades tienen mayores consecuencias ya que van generando la desvinculación productiva femenina, que conlleva a que las entrevistadas que no residen en el campo y no participan de las tareas rutinarias, tengan la "percepción de un conocimiento insuficiente de los procesos productivos", con consecuencias sobre la administración, organización y control de las tareas y de la percepción social de la propiedad femenina.

Sólo una de las entrevistadas que denota alto grado de empoderamiento, crea sus propias reglas y desnaturaliza los constructos sociales del sector. Ella utiliza principalmente estrategias transformacionales generando su espacio, eligió no adaptarse sino por el contrario que se adapten, aclara las reglas de inmediato y si no recibe el trato que esperaba se desvincula inmediatamente. Claramente, ha tenido importantes logros en materia productiva y el reconocimiento de sus pares, al considerarla una pionera en la aplicación de determinadas estrategias de producción. Las restantes mujeres, si bien han optado por capacitación, lectura y asesoramiento como herramienta de conocimiento, no han podido sentirse equiparadas en este aspecto a sus pares varones, sintiendo que las actividades de capacitación son complementarias, 
pero no sustituyen la presencia en el campo y que resultaría central para este conocimiento el mantener la vinculación productiva.

Debe tenerse en cuenta las limitaciones que presenta este trabajo, dado que por tratarse de un estudio de casos los resultados no son generalizables siendo las conclusiones aplicables solo a los casos estudiados, no obstante es importante el aporte pues permite analizar en profundidad la percepción de las barreras o dificultades que sienten aquellas mujeres que decidieron incursionar en una actividad en las que no es habitual que las mujeres se hagan cargo. En tal sentido, resultaría pertinente contar con testimonios de aquellas mujeres que habiendo tenido la posibilidad de la gestión de una empresa de este tipo, decidieron salir de la actividad y conocer las razones de tal decisión.

\section{REFERÊNCIAS BIBLIOGRÁFICAS}

Archetti, E. P. (1977). El proceso de capitalización de campesinos argentinos. Cahiers $d u$ monde hispanique et luso-brésilien, 28 (1)123-140.

Altamirano, H., Macarrein, O., Silva, N. y Paredes, H. (2009); "Modelos productivos ganaderos en la provincia de Corrientes”. Facultad de Ciencias Agrarias, UNNE, 2009.

Babcock, L., \& Laschever, S. (2009). Women don't ask: Negotiation and the gender divide. New Jersey. Princeton University Press.

Barberá Heredia, E., Ramos López, A. \& Candela Agulló, C. (2011) Laberinto de cristal en el liderazgo de las mujeres. Psicothema, 23 (2), 173-179.

Becker, G. S., \& de Grado, C. P. (1987). Tratado sobre la familia (Vol. 1). Madrid. Alianza editorial.

Bordieu P, (2000) La dominación masculina $2^{\text {a }}$ Ed., traducido por Joaguín Jordá. Madrid. Anagrama.

Bryant, G. (1984). The Working woman report: Succeeding in business in the 80s. New York, N.Y: Simon and Schuster.

Creswell, John W, Vicki L Plano Clark, Michelle L Gutmann, And William E Hanson (2003) Advanced Mixed Methods Research Designs en Handbook of Mixed Methods in Social y Behavioral Research: 209-240. CA Sage. Thousand Oaks. 
Do, Q.-T., Levchenko, A. A., \& Raddatz, C. E. (2011). Engendering trade. World Bank. Policy Research Working Paper, (5777).

Eagly, A. H., \& Carli, L. L. (2003a). The female leadership advantage: An evaluation of the evidence. The Leadership Quarterly, 14(6), 807-834. http://www.sciencedirect.com/science/ article/pii/S1048984303000584

Eagly, A. H., \& Carli, L. L. (2003b). Finding gender advantage and disadvantage: Systematic research integration is the solution. The Leadership Quarterly, 14(6), 851-859. http://www. sciencedirect.com/science/article/pii/S1048984303000572

Echaniz, A. (2001). La situación de la mujer en la empresa.: hacia el liderazgo femenino. Caso de MCC. Tesis Doctoral. Universidad de DEUSTO, Campo de San Sebastián.

Elborgh-Woytek, K., Newiak, M., Kochhar, K., Fabrizio, S., Kpodar, K,P., Wingender, P., Clements, B.\& Schwartz, G. (2013). Las mujeres, el trabajo y la economía: Beneficios macroeconómicos de la equidad de género. Documento de análisis del personal técnico del FMI. Disponible http://www.igualdadenlaempresa.es/enlaces/webgrafia/docs/Las_mujeres_ el_trabajo_y-FMI.pdf (consulta 29 de febrero 2016).

Ferber, M. y J. Nelson (Eds.) (2003). Feminist economics today: Beyond Economic Man. Chicago-Londres: The University of Chicago Press.

Ferro, S. L. (2008.). Género y agricultura familiar capitalizada Argentina (19702000).

Ferro, S. L., de Planeamiento, S., \& de Diputados, C. (2007). Agricultura familiar capitalizada en la Argentina reciente. Reduccionismo conceptual neoclásico y alternativas epistemológicas integradoras. In Simposio (No. 6).

Filson, G. C. (1993). Comparative differences in Ontario farmers' environmental atti-tudes. Journal of Agricultural and Environmental Ethics, 6(2), 165-184.

Gailhard, İ. U., \& Bojnec, Š. (2015). Farm size and participation in agri-environmental measures: Farm-level evidence from Slovenia. Land Use Policy, 46, 273-282.

García Prince, E., Kaimowitz, D., Vejarano, M., Ahmad, Y., Festinger, L., Fonseca Zamora, J; \& López-Mora, A. (1993). La Mujer venezolana en el proceso de toma de decisiones: 
situación y perspectivas. (No. 0253-4746). Presidencia de la República, Caracas (Venezuela). Comisión Femenina Asesora.

García-Retamero, R., \& López-Zafra, E. (2006). Congruencia de rol de género y liderazgo: el papel de las atribuciones causales sobre el éxito y el fracaso. Revista Latinoamericana de Psicología, 38(2), 245-257.

Gatica, C. C., \& Rojas, P. S. (2004). Estudios de gerencia y género y su relación con la formación profesional universitaria. Revele Abril-Junio 3(79), 209-226.

Gasson, R., \& Winter, M. (1992). Gender relations and farm household pluriactivity. Journal of Rural Studies, 8(4), 387-397.

Godoy, L., \& Mladinic, A. (2009). Estereotipos y roles de género en la evaluación labo-ral y personal de hombres y mujeres en cargos de dirección. Psykhe (Santiago), 18(2), 51-64.

Guirado, C., \& Luque, I. N. M. \& Molero Alonso, F. (2006). Mujeres y Liderazgo. Claves Psicosociales del Techo de Cristal. Madrid, Sanz y Torres.

Hallward-Driemeier, M., Hasan, T., Kamangu, J., Lobti, E., \& Blackden, M. (2013). Women's Legal and Economic Empowerment Database (Women LEED Africa). Development Economics, Washington, DC.

Haslam, S. A., \& Ryan, M. K. (2008). The road to the glass cliff: Differences in the perceived suitability of men and women for leadership positions in succeeding and failing organizations. The Leadership Quarterly, 19(5), 530-546. http://psycnet.apa.org/index.cfm?fa=buy. optionToBuy\&id=2010-26139-001

Heller, L. (2004). Nuevas voces del liderazgo: Dilemas y estrategias de las mujeres que trabajan. Buenos Aires, Editorial Nuevo Hacer.

Hymowitz, C., \& Schellhardt, T. D. (1986). The glass ceiling: Why women can't seem to break the invisible barrier that blocks them from the top jobs. The Wall Street Journal, 24, 1.

Macarrein, O. F., Almirón, L. R., Altamirano, H. H., \& Paredes, H. G. (2006). La evolución ganadera de la provincia de Corrientes. Universidad Nacional del Nordeste. Comunica- 
ciones Científicas y Tecnológicas, Corrientes.

Navarro, M., \& Korrol, V. S. (2004). Mujeres en América latina y el Caribe. Madrid, Narcea Ediciones.

Paulilo, M. I. S. (2006). El género y la clase en los movimientos de mujeres agricultoras de Brasil. Agricultura, Sociedad y Desarrollo, 3(2), 175-196.

Pérez, M. S. T., Cepeda, M. J. D., del Val Valdivieso, M. I., \& De la Rosa Cubo, C. (2004). La historia de las mujeres: una revisión historiográfica. Secretariado de Publicaciones e Intercambio Editorial.

Perona, E. (2012a). Economía feminista: ensayos sobre el papel de la mujer en la economía, la educación y el desarrollo. Asociación Cooperadora de la Facultad de Ciencias Económicas de la Universidad Nacional de Córdoba.

Perona, E. (2012b). La transformación tecnológica del sector agropecuario en la provincia de Córdoba y sus repercusiones sobre la mujer y la familia rural. Estudios Feministas, 739-760. Córdoba.

Preda, G. (2010). Relaciones de parentesco en la producción familiar capitalizada del sur de Santa Fe. Avá, (16), 1-1. Provoste Fernández, P. (2012). Protección social y redistribución del cuidado en América Latina y el Caribe: el ancho de las políticas. Santiago de Chile, CEPAL.

Ramos López, M. A., Barbera Heredia, E., \& Sarrió Catalá, M. (2003). Mujeres directivas, espacio de poder y relaciones de género. Anuario de Psicología, 34 (2), 267-278.

Ryan, M. K., \& Haslam, S. A. (2005). The glass cliff: Evidence that women are over-represented in precarious leadership positions. British Journal of Management, 16 (2), 81-90.

Schein, V. E. (2001). A global look at psychological barriers to women's progress in management. Journal of Social Issues, 57(4), 675-688.

Traoré, N., Landry, R., \& Amara, N. (1998). On-farm adoption of conservation practices: the role of farm and farmer characteristics, perceptions, and health hazards. Land Economics, $114-127$. 
Zabludovsky, G., \& de Avelar, S. (2001). Empresarias y ejecutivas en México y Brasil. Miguel Ángel Porrúa. Unam. México DF.

\section{CURRICULUM VITAE}

\section{Ludmila Vargas Viola}

Licenciada en Economía, Facultad de Ciencias Económicas, Universidad Nacional de Cuyo (UNCUYO) Argentina, 2003. Especialista en Gestión de Empresas agropecuarias. Universidad Nacional del Nordeste, Argentina, 2015. Diplomada en Desarrollo Humano, Facultad Latinoamericana de Ciencias Sociales, Buenos Aires, Argentina, 2015. Especialista en Gestión y Control de Organizaciones del Sector Público, Universidad de la Cuenca del Plata, Corrientes, Argentina, 2011. Directora Observatorio Social y Económico Facultad de Ciencias Empresariales. Universidad de la Cuenca del Plata. Profesora asociada de Economía y Macroeconomía Facultad Ciencias Empresariales. Universidad de la Cuenca del Plata.

vargasviolamaria_goy@ucp.edu.ar

\section{Patricia Laura Rábago}

Contador Público, Facultad de Ciencias Económicas, Universidad Nacional del Centro (UNICEN) Argentina, 1992. Master en Desarrollo Económico en América Latina, Universidad Internacional de Andalucía. Sevilla, España, 2002. Magíster en Comercialización, UNICEN, Facultad de Ciencias Económicas y Universidad de Federal de Rio Grande do Sul, Brasil, 1998. Investigadora Full Time del Centro de Estudios en Administración. Facultad de Ciencias Económicas. UNICEN. Profesor del Área Académica Economía. Categoría SPU: IV Facultad de Ciencias Económicas. UNICEN.

rabago@econ.unicen.edu.ar 